\title{
Original
}

\section{Relationship between Biological Apatite Alignment and Hemi-occlusion in Rabbit Mandibular Cortical bone}

\author{
Takahiro Ogai, ${ }^{1,2)}$, Toshiyuki Morioka, ${ }^{1,3)}$, Satoru Matsunaga, ${ }^{1,4)}$ Kunihiko Nojima2), \\ Yasushi Nishiii ${ }^{2}$, Kenji Sueishii' ${ }^{2)}$ and Masao Yoshinari ${ }^{1)}$ \\ 1) Division of Oral Implants Research, Oral Health Science Center, Tokyo Dental College,Chiba, Japan \\ 2) Departments of Orthodontics, Tokyo Dental College,Chiba, Japan \\ 3) Departments of Oral and Maxillofacial Implantology, Tokyo Dental College, Chiba, Japan \\ 4) Departments of Anatomy, Tokyo Dental College,Chiba, Japan \\ (Accepted for publication, Februrary 20, 2012)
}

\begin{abstract}
Biological apatite (BAp) crystallite c-axis alignment is known to one of the factor in mechanical function in bone. The purpose of this study was to investigate BAp crystallite alignment as a potential factor in the evaluation of bone strength in clinical practice by evaluating the relationship between BAp alignment and bone mineral density (BMD) using an experimental hemi-occlusion model in rabbit mandibular cortical bone.

A higher degree of BAp crystallite c-axis alignment was observed in the mesiodistal direction on the nonocclusion than on the occlusion side in the alveolar area, a tendency that was not seen in the base of the mandible. No significant differences were observed in BMD between the alveolar area and the base of the mandible or between the occlusion and non-occlusion sides. No correlation was observed between BAp crystallite alignment and BMD.

These results demonstrate that removal of occlusal force caused change in BAp crystallite alignment in the alveolar area in mandibular cortical bone. This indicates the importance of evaluating BAp crystallite alignment in addition to BMD in clinical practice. Moreover, clarification of BAp crystallite alignment in the jawbone would allow direction of occlusal load to be taken into account in orthodontic treatment involving tooth alignment.
\end{abstract}

Keywords: Hemi-occlusion, Bone quality, Biological apatite, Crystallite alignment, Bone mineral density

\section{Introduction}

The mandible is exposed to direct stress on occlusion of the teeth and indirect stress via the masticatory muscles and soft tissue during mastication. Long-term masticatory disturbances caused by abnormal occlusion or abnormal functioning may therefore alter the shape and inner structure of the mandible. Tooth grinding or tooth extraction can change the magnitude of mechanical stimuli, thus affecting the strength and elastic modulus of bone and trabecular structure. ${ }^{1,2)}$ The direction and magnitude of mechanical stress are difficult to measure, so predictions of mechanical stress have largely been based on simulation analyses. ${ }^{3)}$ The most commonly used indicator for evaluation of bone is the bone mineral density (BMD) index, because direct measurement of the influence of mechanical stimuli generated in vivo on bone is difficult. Moreover, the BMD has been reported to show a high correlation with bone strength. ${ }^{4)}$ However, determination of the $\mathrm{BMD}$ alone is insufficient for accurate evaluation of bone

Correspondence to: Dr. Masao Yoshinari, Division of Oral Implants Research, Oral Health Science Center,Tokyo Dental College1-2-2 Masago, Mihama, Chiba 261-8502, Japan. E-mail:yosinari@tdc.ac.jp, Phone: +8143-270-3536, Fax: +81-43-270-3780 strength. ${ }^{5)}$

At the NIH consensus development conference on osteoporosis held in 2000, bone quality was proposed as a potential indicator of bone strength where the BMD alone was insufficient. ${ }^{6}$ Among the parameters proposed, the alignment of biological apatite (BAp) crystallites, in particular, attracted interest. C-axis alignment of BAp crystallites along the c-axis in collagen fibers is closely related to the mechanical function of bone. A strong correlation has been reported between alignment of BAp crystallites in the main direction of stress and bone strength. ${ }^{7-12)}$

Using a microbeam X-ray diffractometer system, Nakano et al. were able to measure BAp crystallite alignment over even a small region. ${ }^{13)}$ In this system, a collimator is used to narrow the $\mathrm{X}$-ray beam to as little as 10 to $100 \mu \mathrm{m}$, enabling quantitative evaluation of BAp crystallite alignment in microscopic regions of the mandible. In an earlier study, Koizumi et al. investigated the effect of masticatory laterality on the morphology and internal structure of the mandible using experimental hemi-occlusion in growing rabbits and showed that mechanical stimuli affected the morphology and architecture of trabecular bone in rabbit mandible 


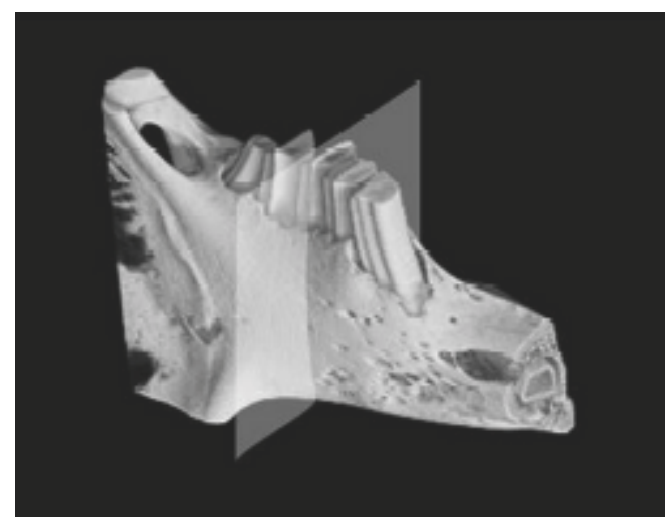

Figure 1. Stereoscopic model and slice image of ROI (mandibular third molar)

during the growth period. ${ }^{2)}$ However, the relationship between bone quality, including BAp crystallite alignment, and the microarchitecture of cortical bone remains to be clarified.

The purpose of this study was to investigate BAp crystallite alignment as a potential factor in the evaluation of bone strength in clinical practice using an experimental hemi-occlusion model in rabbit.

\section{Materials and Methods}

\section{Experimental methods}

Six, 5-week-old (747 \pm 64.7 g each), male, Japanese, white rabbits (Sankyo Labo Service Corporation Inc., Tokyo, Japan) were used in this experiment. All animals were fed a solid diet.

After placing the rabbits under general anesthesia with $3 \%$ to $4 \%$ isoflurane, the left maxillary and mandibular molars were cut as far as the cervical area using a rabbit molar cutter (PROMICLOS Corporation, Tokyo, Japan), taking care not to expose the pulp. Under these conditions of experimental hemi-occlusion, the right side was defined as the occlusion side and the left side as the nonocclusion side Thereafter, the teeth were cut once every 2 weeks and the rabbits kept until they were 17 weeks old (approximately 3000 g each).

After this designated period, the rabbits were euthanized with an overdose of sodium thiopental administered intravenously. The rabbits were then decapitated and the mandibles extracted after resection of soft tissue. All procedures were performed in accordance with the Guidelines for Animal Experiments at Tokyo Dental College (Approval Number 222805) and the Helsinki Declaration, ensuring that no rabbit experienced pain. This procedure was the same as that described by Koizumi et al.2)

\section{Region of interest and measurement sites}

Images of the mandible were obtained using micro-CT (HMX225-ACTIS, Tesco Corporation, Tokyo, Japan). Micro-CT was performed with a tube voltage of $130 \mathrm{kV}$, tube current of 80 $\mu \mathrm{A}$, magnification of 4.62, and a slice width of $50 \mu \mathrm{m}$.

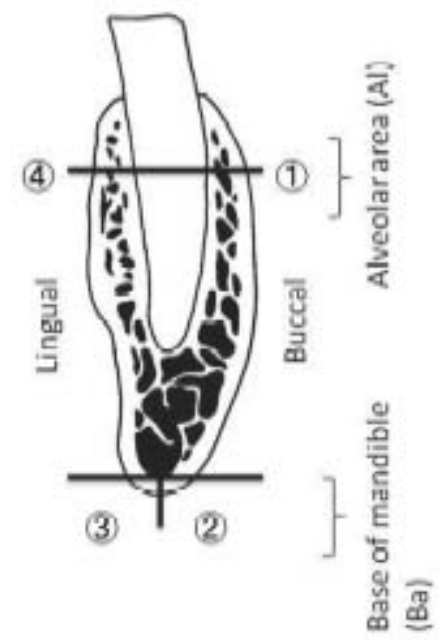

Figure2. Schematic of measurement sites.

Measurement sites in alveolar area (Al: Buccal side (1) Lingual side: (4)), Measurement sites at base of mandible (Ba: Buccal side (2), Lingual side (3))

A 3-dimensional (3-D) structural analysis software (TRI/3-DBON, Ratoc System Engineering Corporation Ltd., Tokyo, Japan) was used to reconstruct 3-D images from image data obtained by micro-CT. The middle section of the first postmolar was marked as the region of interest (ROI, Fig. 1). Measurements were performed at a total of 4 sites in each specimen (Fig. 2): at (1) on the buccal side of the alveolar area (Al); at (2) at the base of the mandible ( $\mathrm{Ba}$ ) on the buccal side; at (3) at $\mathrm{Ba}$ on the lingual side; and at (4) on the lingual side of $\mathrm{Al}$. Regions $3 \mathrm{~mm}$ from the alveolar crest on the (1) buccal side of $\mathrm{Al}$ and (4) lingual side of $\mathrm{Al}$ were measured. The area under the $\mathrm{Ba}$ cancellous bone was bisected to create equal buccal and lingual sides, and points on $\mathrm{Ba}$ on the (2) buccal side and (3) lingual side were designated as measurement sites. Sites (1) and (4) represented Al and sites (2) and (3) represented $\mathrm{Ba}$. In the evaluations, the measurement sites were not divided into the buccal and lingual sides, but considered as belonging solely to $\mathrm{Al}$ or $\mathrm{Ba}$, as the results of an earlier experiment showed that there were no differences in BAp alignment between the buccal and lingual sides. ${ }^{14)}$

\section{Determination of BAp crystallite alignment}

After embedding in epoxy resin, the specimens were cut buccolingual direction with a diamond cutter and polished with a series of SiC papers of \#400, \#800, and \#1200 using a polishing machine (ECOMET3, Buehler, Germany). A microbeam X-ray diffractometer (RINT2500, Rigaku Corporation, Tokyo, Japan) was used for the X-ray diffraction analysis. Measurement was carried out using $\mathrm{Cu}-\mathrm{K} \alpha$ beams with a voltage of $40 \mathrm{kV}$ and current of $200 \mathrm{~mA}$. The incident beam was focused on a spot $100 \mu \mathrm{m}$ in diameter using a collimator. To obtain the averaged diffraction data within a particular azimuth range, oscillation was carried out under the following conditions: $\omega, 17.3^{\circ} \pm 5.5^{\circ}$ (step width: $1.1^{\circ}$ ), 
Takahiro Ogai et al.,: Biological Apatite Crystallite Alignment in Hemi-Occlusion
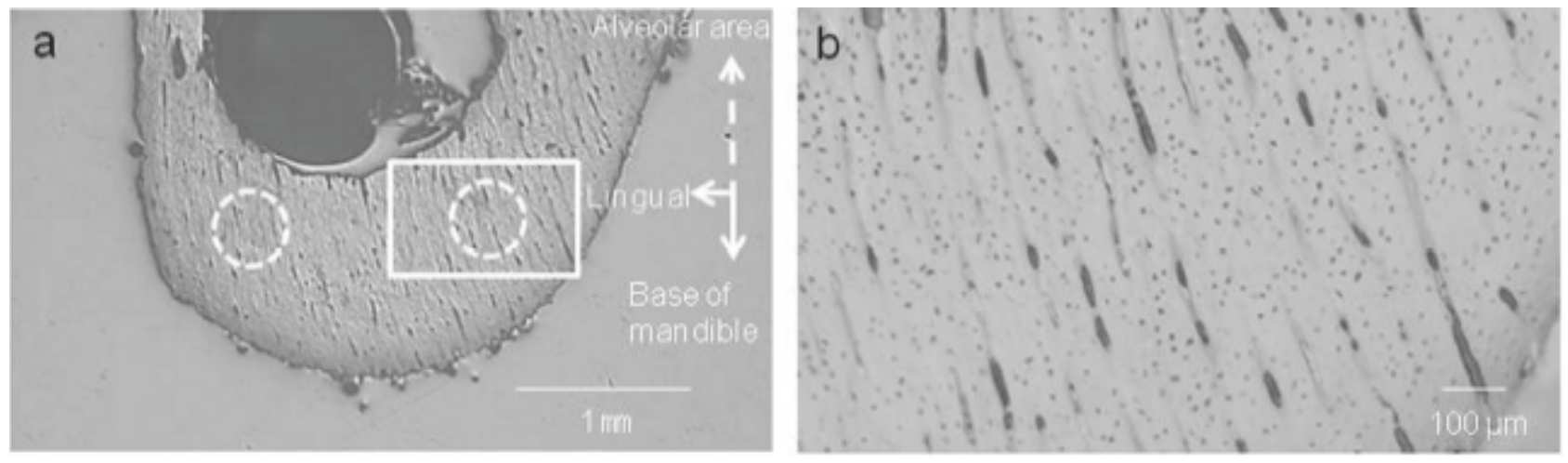

Figure 3. Base of mandible from specimen used for determination of BAp crystallite alignment (a: Lower magnification; b: Higher magnification) View b represents magnification of boxed area in view a.

XRD measurements were performed at different 3 points within each selected area shown in dotted circles in Figure $3 a$.

$\chi, 0^{\circ} \pm 0.6^{\circ}$ (step width: $0.3^{\circ}$ ), and $\varphi, 180^{\circ} \pm 180^{\circ}$ (continuous). This diameter was sufficiently smaller than analytical area of region of interest (Fig. 2) even though under the fluctuations $\left(17.3^{\circ}\right.$ $\pm 5.5^{\circ}$ ) during XRD measurement. Diffracted X-rays were recorded with a curved position sensitive proportional X-ray counter.

Figure 3 shows a typical example of the location at which the base of the specimen mandible was measured. Fig. 3b represents a magnification of the boxed area in Fig. 3a. In order to determine BAp crystallite alignment, we used a CCD camera equipped with an optical microscope to enlarge the specimen, focusing on the area between the external and internal circumferential lamellae of the cortical bone. X-ray diffraction (XRD) measurements were performed at 3 different points within each of the selected areas shown in dotted circles in Fig. 3a.

Biological apatite crystallite alignment was evaluated by calculating the ratio $\{\mathrm{I}(002) / \mathrm{I}(310)\}$ of the integrated intensity of the (002) surface (in the vicinity of $2 \theta=25.9^{\circ}$ ) of the XRD profile to that of the (310) surface (in the vicinity of $2 \theta=39.8^{\circ}$ ). Increase in the intensity ratio indicated an increase in c-axis alignment of the BAp crystallites. Hydroxyapatite (HAp) particles with no crystal alignment (Wako Pure Chemical Industries, Ltd., Japan) were used as a control. The (002)/(310) intensity ratio of the HAp particles as an indicator with no alignment was 0.74 .

\section{Bone mineral density (BMD) measurement}

Images were taken of phantoms with various layers of density (200 to $800 \mathrm{mg} / \mathrm{cm}^{3}, \varphi 6 \times 1 \mathrm{~mm}$ ), and a standard curve was created between the $\mu \mathrm{CT}$ data obtained and existing phantom densities. A 3-D trabecular structure measurement software (TRI/3D-BONBMD-PNTM2, RATOC System Engineering, Japan) was then used to convert the mandible $\mu \mathrm{CT}$ image data into BMD values and create color labeled images. Slice images were created at 1mm widths in the ROIs of the converted BMD images, and cortical bones were selected by means of binarization. Measurement was carried out on the occlusion and non-occlusion sides at each of sites (1) to (4) in the same way as in X-ray diffraction analysis. 5. Statistical analysis

A two-way analysis of variance (ANOVA) was performed with differences in occlusal force (between the occlusion and nonocclusion sides) as factor A and structural differences in the mandible (between $\mathrm{Al}$ and $\mathrm{Ba}$ ) as factor B. Subsequently, Scheffé's multiple comparisons were performed. A value of $p<0.05$ was considered statistically significant.

\section{Results}

\section{BAp crystallite alignment}

Figure 4 shows typical X-ray profiles obtained from rabbit mandible (a: Al/Occlusion side; b: Al/Non-occlusion side; c: $\mathrm{Ba} /$ Occlusion side; d: Ba/Non-occlusion side). Larger intensity (002)/ (310) ratios were observed at $\mathrm{Ba}$ on both the occlusion and nonocclusion (c, d) sides than in $\mathrm{Al}$ (a,b). In addition, larger intensity (002)/(310) ratios were observed on the non-occlusion side (b) than on the occlusion side (a).

Table 1 shows the results of the two-way ANOVA on BAp crystallite alignment with differences in occlusal force designated as factor A and structural differences in the mandible as factor B. Highly significant differences were found between levels for both factor A (differences in occlusal force; $p<0.05$ ) and factor B (structural differences in the mandible; $p<0.01$ ).

Figure 5 shows BAp crystallite alignment categorized by differences in occlusal force (occlusion and non-occlusion sides) and structural differences in mandible (Al: (1) and (4), Ba: (2) and (3)). The (002)/(310) intensity ratios at all sites were larger than those of the HAp particles with no crystal alignment used as a control (0.74), and the results indicated preferential BAp crystallite alignment along the longitudinal axis (mesiodistal direction) of the mandible. Intensities were larger at Ba than in $\mathrm{Al}$, and within $\mathrm{Al}$, removal of occlusal force resulted in greater intensities on the non-occlusion than on the occlusion side.

These results demonstrated that the degree of BAp crystallite alignment along the longitudinal axis of the rabbit mandible was 

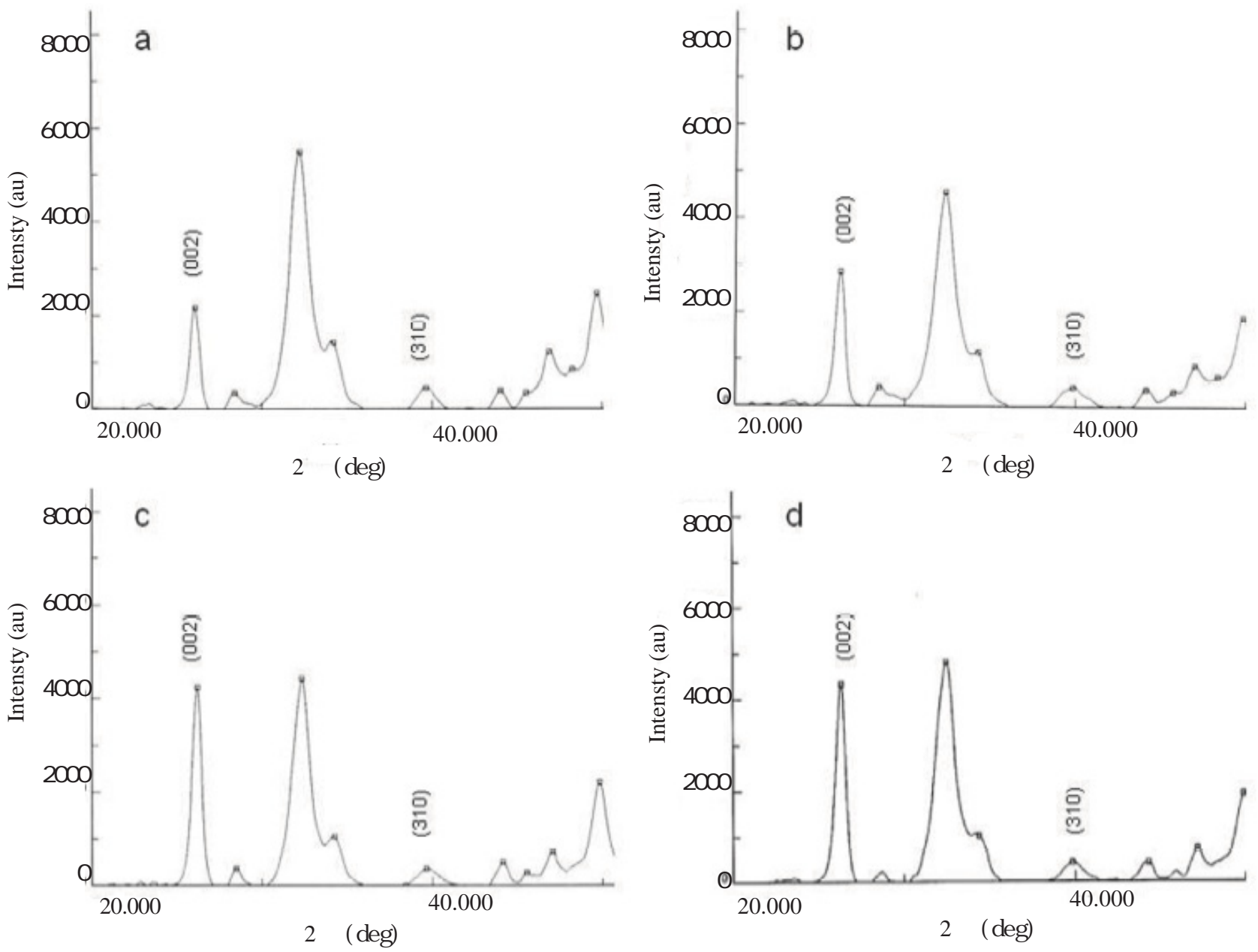

Figure 4. Typical X-ray profiles of rabbit mandible (a: Al/Occlusion side; b: Al/Non-occlusion side; c: Ba/Occlusion side; d: Ba/Non-occlusion side)

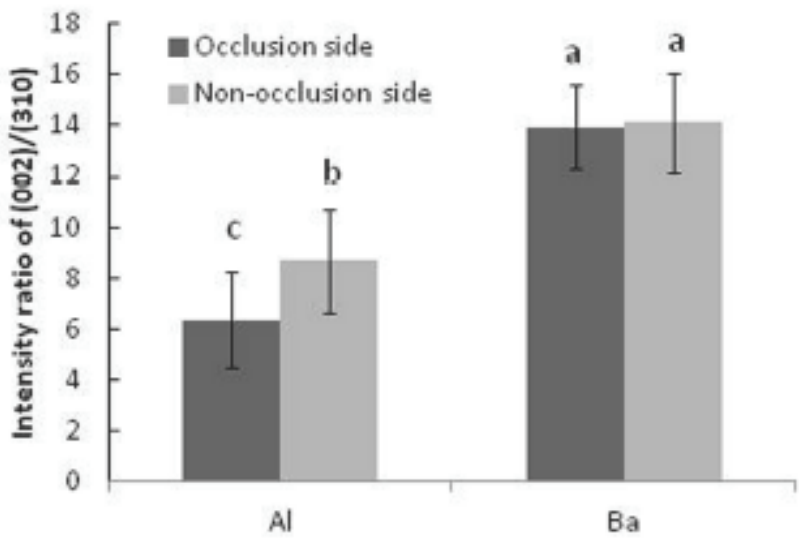

Figure 5. BAp crystallite alignment categorized by structural differences (Al, Ba) and differences in occlusal force (occlusion and non-occlusion sides). Same letters indicate no significant difference; different letters indicate significant difference.

higher at $\mathrm{Ba}$ than in $\mathrm{Al}$, and that removal of occlusal force affected BAp crystallite alignment in the alveolar area.

\section{2. $B M D$}

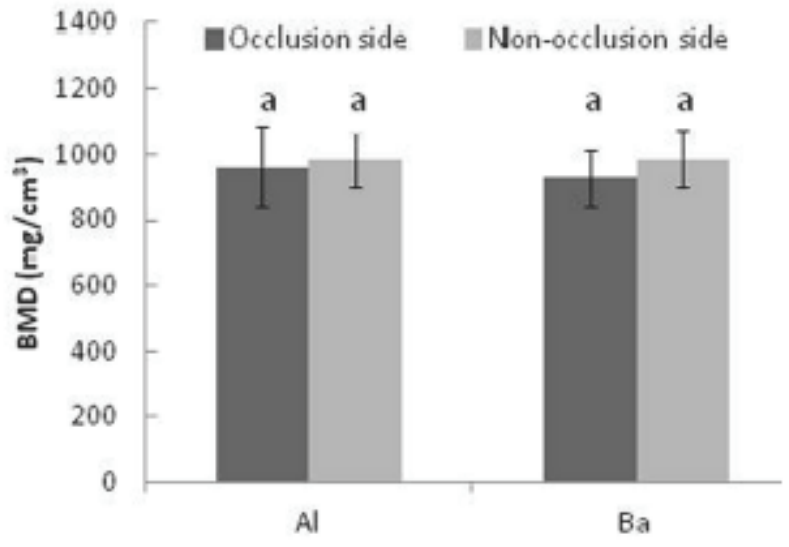

Figure 6. BMD values categorized by structural differences (Al, Ba) and differences in occlusal force (occlusion and non-occlusion sides). Same letters indicate no significant difference.

Table 2 shows the results of the two-way ANOVA on BMD with differences in occlusal force designated as factor $\mathrm{A}$ and structural differences in the mandible as factor B. No significant 
Takahiro Ogai et al.,: Biological Apatite Crystallite Alignment in Hemi-Occlusion

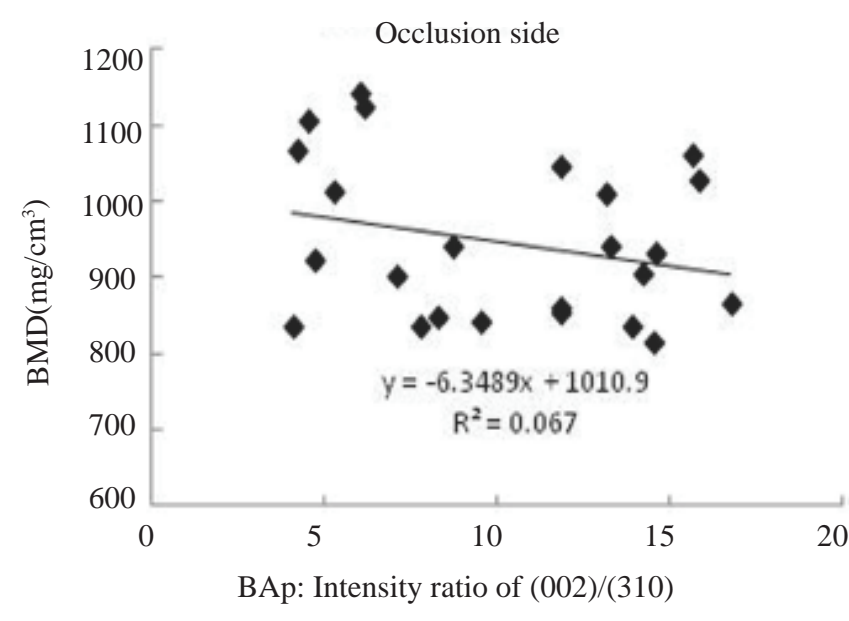

Figure 7. Diagram showing correlation between BAp crystallite alignment and BMD values (occlusion side)

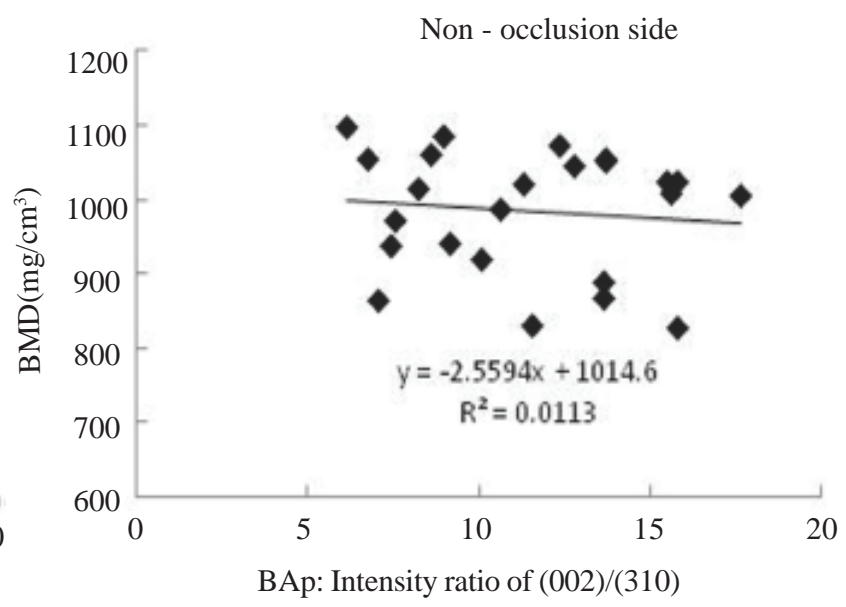

Figure 8. Diagram showing correlation between BAp crystallite alignment and BMD values (non-occlusion side)

Table 1. Results of two-way ANOVA on BAp variance with differences in occlusal force as factor A (occlusion and non-occlusion sides) and structural differences as factor B (A1, Ba).

\begin{tabular}{lcccrcc}
\hline Factor & Sum of squares & Degree of freedom & Mean square & F value & P value & Judgment \\
\hline Factor A & 18.5335 & 1 & 18.5 & 5.17 & 0.0279 & $*$ \\
Factor B & 508.2375 & 1 & 508.2 & 141.79 & 0.0000 & $* *$ \\
Factor A*Factor B & 13.8536 & 1 & 13.9 & 3.87 & 0.0556 & \\
Error & 157.7126 & 44 & 3.6 & & & \\
Overall & 698.3373 & 47 & & & & \\
\hline
\end{tabular}

Significant difference: $* \mathrm{P}<0.05,{ }^{* *} \mathrm{P}<0.01$

Table 2. Results of two-way ANOVA on BMD with differences in occlusal force as factor A (occlusion and non-occlusion sides) and structural differences as factor $\mathrm{B}(\mathrm{A} 1, \mathrm{Ba})$

\begin{tabular}{lcccccc}
\hline Factor & Sum of squares & Degree of freedom & Mean square & F value & P value & Judgment \\
\hline Factor A & 18240.3019 & 1 & 18240.3 & 2.03 & 0.1613 & \\
Factor B & 2850.5419 & 1 & 2850.5 & 0.32 & 0.5762 & \\
Factor A*Factor B & 4830.0469 & 1 & 4830.0 & 0.54 & 0.4674 & \\
Error & 395436.7725 & 44 & 8987.2 & & & \\
Overall & 421357.6631 & 47 & & & & \\
\hline
\end{tabular}

differences were observed for either factor A or factor B ( $p>0.05)$. Figure 6 shows BMD values categorized by structural differences in the mandible (Al: (1) and (4), Ba: (2) and (3)) and differences in occlusal force (between occlusion and non-occlusion sides). No significant differences were observed between each level $(p>0.05)$.

\section{Relationship between BAp crystallite alignment and BMD}

Figures 7 and 8 show the relationship between BAp crystallite alignment and BMD values on the occlusion and non-occlusion side, respectively. No correlation was identified between BAp crystallite alignment and BMD on either the occlusion or nonocclusion sides $(p>0.05)$.

\section{Discussion}

\section{BAp crystallite alignment}

In vivo, BAp crystallites tend to align along the direction of stress. In a study on monkey mandibles, Nakano et al. ${ }^{13)}$ reported that while BAp crystallites were basically aligned in the mesiodistal direction along the longitudinal axis, they aligned in the occlusal direction immediately below the dental crown. Furthermore, in a study on developing rat mandibles, Nakano et 
J.Hard Tissue Biology Vol. 21(2):165-172, 2012

al. ${ }^{15)}$ reported that BAp crystallite alignment in the site immediately below the dental root was influenced by occlusion. The present study also confirmed preferential BAp crystallite alignment along the longitudinal axis of the mandible. In addition, alignment was confirmed to be low in $\mathrm{Al}$ and high at $\mathrm{Ba}$. Similar results were reported in an earlier study by Nakano et al. investigating BAp crystallite alignment in monkey mandible, as mentioned above. ${ }^{13)}$ This suggests that force applied to the teeth affects BAp crystallite alignment in $\mathrm{Al}$ in mandible cortical bone, that is, occlusal force may increase BAp crystallite alignment along the occlusal direction, and decrease BAp alignment along the mesiodistal direction in $\mathrm{Al}$.

In the present study, a higher degree of BAp crystallite alignment was observed along the mesiodistal direction on the non-occlusion side, the side on which occlusal force had been removed. In studies on beagle mandible, Fujitani et al. ${ }^{16)}$ reported that removal of occlusal force led to a change in BAp crystallite alignment at sites immediately beneath the teeth, from twodimensional alignment to one-dimensional alignment. The present results support this finding. Thus, decrease in BAp crystallite alignment in the occlusal direction may have caused the increase in alignment in the mesiodistal direction (one-dimensional direction) observed here.

\section{BMD}

While some reports indicate that BMD decreases after a decline in mechanical stress, ${ }^{17)}$ others indicate a rise in BMD. ${ }^{16)}$ Other reports found no change in BMD with a decline in mechanical stress. ${ }^{18-20)}$ In the present study, no significant differences were found in BMD between the occlusion and non-occlusion sides in either $\mathrm{Al}$ or $\mathrm{Ba}$. This may be because measurements were taken in cortical bone ${ }^{17,21)}$ and no resorption had taken place in $\mathrm{Al}$ as no teeth were extracted. ${ }^{16)}$ and BMD was maintained by functional stress generated by the masticatory muscles. ${ }^{18)}$

\section{Relationship between BAp crystallite alignment and BMD}

The results of the two-way ANOVA revealed significant differences in factor $\mathrm{A}(p<0.05)$, which analyzed differences in BAp crystallite alignment due to differences in occlusal force between the occlusion and non-occlusion sides, but no significant differences in factor A for BMD. In BAp crystallite alignment, significant differences were observed in factor $\mathrm{B}(p<0.01)$, which analyzed differences in BAp crystallite alignment (between $\mathrm{Al}$ and $\mathrm{Ba}$ ) caused by structural differences in the mandible. However, no significant differences were seen in factor B for BMD. These results demonstrate that differences in occlusal force and structural differences in the mandible between $\mathrm{Al}$ and Ba mainly affect BAp alignment.

Examination of the relationship between BAp crystallite alignment and BMD (Figs. 5 and 6) revealed that the BMD values were very similar, but that BAp crystallite alignment was dispersed. Moreover, no correlation was observed between BAp crystallite alignment and BMD values on either the occlusion or non-occlusion sides. These results indicate that BAp crystallite alignment and BMD should be understood as independent factors.

\section{Clinical implications}

The results of the present study suggest that BAp crystallite alignment and BMD values represent different responses to mechanical stress. Furthermore, while previous studies reported a high correlation between BMD and bone strength, ${ }^{3)}$ the present study found no significant differences in BMD between sites in mandibular cortical bone. In contrast, BAp crystallite alignment showed a strong response to mechanical stress that differed significantly between sites. Although BMD can be evaluated using medical imaging modalities such as computed tomography, it is impossible to evaluate bone micro-architecture and thus extremely difficult to evaluate bone quality encompassing bone strength. in a clinical setting. ${ }^{22)}$ This indicates the potential of evaluating bone quality in addition to BMD when determining bone strength in clinical practice. Moreover, clarification of BAp crystallite alignment in the jawbone would allow direction of occlusal load to be taken into account in orthodontic treatment involving tooth alignment.

\section{Acknowledgments}

This research was supported by an Oral Health Science Center Grant HRC7 from Tokyo Dental College, and by a "High-Tech Research Center” Project for Private Universities: matching fund subsidy from MEXT (Ministry of Education, Culture, Sports, Science and Technology) of Japan, 2006-2010. The authors would like to thank Associate Professor Jeremy Williams, Tokyo Dental College, for his assistance with the English of the manuscript..

\section{References}

1. Wada H, Hosomichi J, Shimomoto Y and Soma K. Influence of occlusal hypofunction on the elastic property and bone formation of rat alveolar bone. Orthodontic Wave 67: 9-14, 2008

2. Koizumi Y, Ishii T, Nishii Y, Nojima K and Sueishi K. Influence of experimental hemi-occlusion on mandibular morphology and internal structure in growing rabbit. Orthodontic Wave 69: 58-65, 2010

3. Ichim I, Kieser JA and Swain MV. Functional significance of strain distribution in the human mandible under masticatory load: Numerical predictions. Arch Oral Biol 52: 465-473, 2007

4. Snyder BD, Piazza S, Edwards WT and Hayes WC. Role of trabecular morphology in the etiology of age-relatedvertebral fractures. Calcif Tissue Int 53: 14-22, 1993 
Takahiro Ogai et al.,: Biological Apatite Crystallite Alignment in Hemi-Occlusion

5. Dougherty G. Quantitative CT in the measurement of bone quantity and bone quality for assessing osteoporosis. Med Eng Phys 18: 557-568, 1996

6. NIH Consensus Development Panel on Osteoporosis Prevention, Diagnosis, and Therapy. JAMA 285: 785-795, 2001

7. Elliot JC. Structure and chemistry of the apatites and other calcium orthophosphates. Amsterdam: Elsevier, pp 1-389, 1994

8. Daculsi G, Bouler JM and LeGeros RZ. Adaptive crystallite formation in normal and pathological calcifications in synthetic calcium phosphate and related biomaterials. Int Rev Cytol 172: 129-191, 1997

9. Bonfield W and Grynpas MD. Anisotropy of the Young's modulus of bone. Nature 270: 453-454,1977

10. Sasaki N, Matsushima N, Ikawa T, Yamamura H and Fukuda A. Orientation of bone mineral and its role in the anisotropic mechanical properties of bone-transverse anisotropy. J Biomech 22: 157-164, 1989

11. Landis WJ, Song MJ, Leith A, McEwen L and McEwen BF. Mineral and organic matrix interaction in three dimensions by high-voltage electron microscopic tomography and graphic image reconstruction. J Struct Biol 110: 39-54, 1993

12. Nakano T, Ishimoto T, Umakoshi $Y$ and Tabata Y. Texture of biological apatite crystallites and the related mechanical function in regenerated and pathological hard tissues. J Hard Tissue Biology 14: 253-254, 2005

13. Nakano T, Kaibara K, Tabata Y, Nagata N, Enomoto S, Marukawa E and Umakoshi Y. Unique alignment and texture of biological apatite crystallites in typical calcified tissues analyzed by microbeam x-ray diffractometer system. Bone
31: 479-487, 2002

14. Morioka T, Matsunaga S, Yoshinari M, Ide Y, Nakano T, Sekine H, Yajima Y. Alignment of biological apatite crystallites at first molar in human mandible cortical bone, Cranio, 30: 32-40, 2012

15. Nakano T, Fujitani W, Ishimoto T and Umakoshi Y. Adaptation of BAp crystal orientation to stress distribution in rat mandible during bone growth. Journal of Physics 165: 1-6, 2009

16. Fujitani $\mathrm{W}$ and Nakano T. Change in biological apatite alignment in beagle mandible. Materials Science Forum 654656: 2216-2219, 2010

17. Kunii R, Yamaguchi M, Aoki Y, Watanabe A and Kasai K. Effect of experimental occlusal hypofunction,and its recovery,on mandibular bone mineral density in rats. European Journal of Orthodontics 30: 52-56, 2008

18. Klemetti E, Vainio P and Kröger H. Muscle strength and mineral densities in the mandible. Gerodontology 11: 7679, 1994

19. Picard S, Lapointe N, Brown J and Guertin P. Histomorphometric and densitometric changes in the femora of spinal cord transected mice. The Anatomical Record 291: 303-307, 2008

20. Tsai C, Huang R, Lee C, Hsiao W and Yang L. Morphologic and bony structural changes in the mandible after a unilateral injection of botulinum Neurotoxin in adult rats. J Oral Maxillofac Surg 68: 1081-1087, 2010

21. Nomura A. Alveolar bone changes due to loss of masticatory stress. Jpn J Oral Biol 24: 951-977, 1982

22. Donnelly E. Methods for assessing bone quality. Clin Orthop Relat Res 469: 2128-2138, 2011 
J.Hard Tissue Biology Vol. 21(2):165-172, 2012 